Clebsch-Gordan coefficients and related identities obtained by an integration over the group space of $\mathrm{SU}_{\mathrm{q}}(2)$

A. C. Cadavid, and R. J. Finkelstein

Citation: Journal of Mathematical Physics 36, 1912 (1995); doi: 10.1063/1.531094

View online: http://dx.doi.org/10.1063/1.531094

View Table of Contents: http://aip.scitation.org/toc/jmp/36/4

Published by the American Institute of Physics

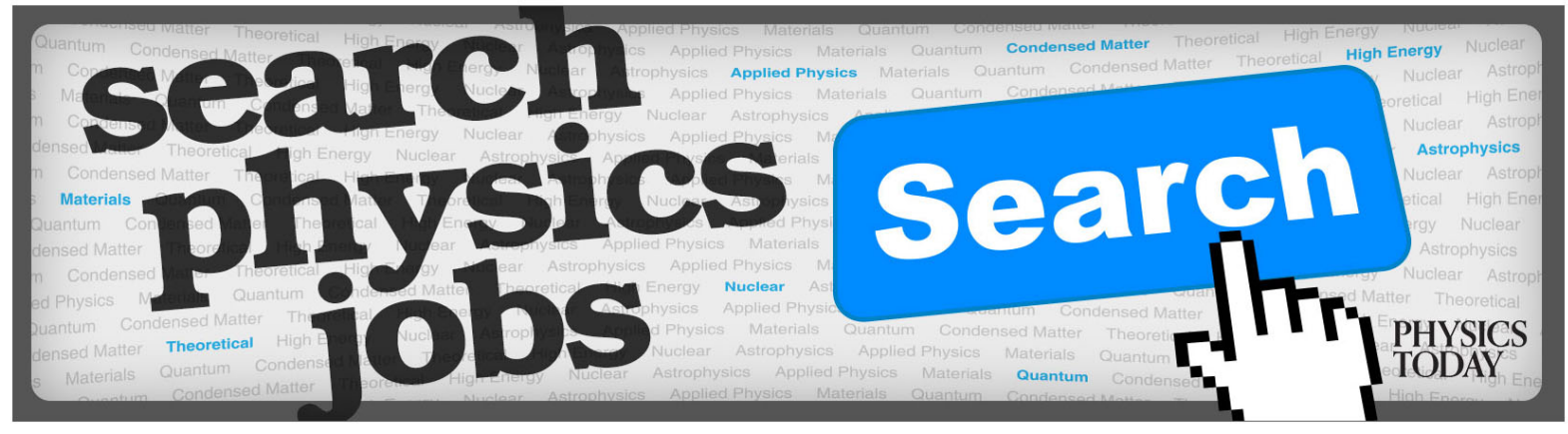




\title{
Clebsch-Gordan coefficients and related identities obtained by an integration over the group space of $\mathrm{SU}_{q}(2)$
}

\author{
A. C. Cadavid \\ Department of Physics and Astronomy, California State University, Northridge, \\ California 91330-8268 \\ R. J. Finkelstein \\ Department of Physics, University of California, Los Angeles, California 90024-1547
}

(Received 8 August 1994; accepted for publication 20 December 1994)

\begin{abstract}
The Clebsch-Gordan coefficients of $\mathrm{SU}_{q}(2)$ are here calculated by an integration over the group manifold following the Woronowicz prescription. A representation is obtained that is different from the one derived from the $q$-group algebra. The equivalency of the two results implies a $q$-identity and establishes a relation between $q$-hypergeometric functions. In the limit $q=1$, our result gives a different expression for the Clebsch-Gordan coefficients of SU(2), and the $q$-identity relation reduces to an identity between binomial coefficients. The Woronowicz technique is extended to calculate the integral of the product of many irreducible representations. A summary of the main results has already been presented elsewhere. (1) 1995 American Institute of Physics.
\end{abstract}

\section{INTRODUCTION}

The Clebsch-Gordan coefficients of the quantum group $\mathrm{SU}_{q}(2)$ have been calculated by several authors, including Kirillov and Reshetikhin, ${ }^{1}$ Groza, Kachurik, and Klimyk, ${ }^{2}$ and Kroelink and Koornwinder. ${ }^{3}$ Although there is no disagreement or ambiguity in their results, we shall obtain the Clebsch-Gordan coefficients by another procedure which leads to apparently different results that nevertheless must be equivalent and must be related to their result by a $q$-identity.

We adapt an early approach by Wigner ${ }^{4}$ which depends on an integration over the group space. Since the $q$-group space is not a continuum, however, the Wigner integration must be replaced by the Woronowicz integration. ${ }^{5}$ We are able to check our results with the cited articles in particular cases, even though the general formulas look quite different. Since we are calculating the same coefficients in different ways, these differently appearing formulas should be related by identities. In the course of our work we also extend the Woronowicz integral over the $q$-group space to the product of many irreducible representations. Finally on descending to the $q=1$ case we obtain alternative presentations for the SU(2) Clebsch-Gordan coefficients.

The work here presented gives details and expands on the results reported in Ref. 6. In Sec. II we establish the notation to be used throughout the article and record the necessary $q$-binomial identities. Section III describes basic $\mathrm{SU}_{q}(2)$ relations and in Sec. IV we introduce the relevant representation and the orthogonal relations obtained via a linear operator related to the Haar measure in the Woronowicz theory. Section V describes the technique to obtain the ClebschGordan coefficients that is used in Sec. VI to calculate some limiting cases. Section VII presents the results for the general coefficients and establishes an identity of $q$-hypergeometric functions by comparing our results with those previously encountered in the literature. In Sec. VIII we explore the consequences of the relations found in Sec. VII for the limiting case $q=1$. Section IX generalizes the techniques of Sec. IV to calculate the integral of a multiple product on $q$-group space. 


\section{II. $q$-BINOMIAL IDENTITIES}

The $q$-binomial theorem is

$$
\begin{aligned}
(A+B)^{n} & =\sum\left\langle\begin{array}{l}
n \\
s
\end{array}\right\rangle_{q} B^{s} A^{n-s}, \\
& =\sum\left\langle\begin{array}{l}
n \\
s
\end{array}\right\rangle_{q_{1}} A^{s} B^{n-s}, \quad q_{1}=q^{-1},
\end{aligned}
$$

where

$$
\begin{gathered}
A B=q B A, \\
\left\langle\begin{array}{l}
n \\
s
\end{array}\right\rangle_{q}=\frac{\langle n\rangle_{q} !}{\langle s\rangle_{q} !\langle n-s\rangle_{q} !}
\end{gathered}
$$

and

$$
\langle n\rangle_{q}=\frac{q^{n}-1}{q-1}
$$

$\langle n\rangle_{q}$ is known as a basic number.

From Eqs. (2.1) and (2.3) we have

$$
(A+B)^{n}(A+B)^{m}=\sum\left\langle\begin{array}{c}
n \\
s
\end{array}\right\rangle\left\langle\begin{array}{c}
m \\
t
\end{array}\right\rangle B^{s} A^{n-s} B^{t} A^{m-t}=\sum\left\langle\begin{array}{c}
n \\
s
\end{array}\right\rangle\left\langle\begin{array}{c}
m \\
t
\end{array}\right)^{s \cdot t} A^{n+m-s-t} q^{(n-s) t}
$$

But

$$
(A+B)^{n}(A+B)^{m}=(A+B)^{n+m}=\sum\left(\begin{array}{c}
m+n \\
u
\end{array}\right) B^{u} A^{m+n-u},
$$

where $q$ is to be understood if the subscript on \langle\rangle is omitted. It follows that

$$
\left\langle\begin{array}{c}
n+m \\
u
\end{array}\right\rangle=\sum_{s=0}^{n}\left\langle\begin{array}{c}
n \\
s
\end{array}\right\rangle\left\langle\begin{array}{c}
m \\
u-s
\end{array}\right\rangle q^{(n-s)(u-s)}
$$

Note also

$$
\langle-n\rangle=\frac{q^{-n}-1}{q-1}=-q^{-n}\langle n\rangle .
$$

The preceding relation permits a useful transformation of $\left\langle\begin{array}{l}n \\ s\end{array}\right\rangle$ $\left\langle\begin{array}{l}n \\ s\end{array}\right\rangle=\langle n\rangle\langle n-1\rangle \cdots\langle n-s+1\rangle /\langle s\rangle !=(-)^{s} q^{n}\langle-n\rangle q^{n-1}\langle-n+1\rangle \cdots q^{n-(s-1)}\langle-n+s-1\rangle /\langle s\rangle !$ or

$$
\left\langle\begin{array}{l}
n \\
s
\end{array}\right\rangle=(-)^{s} q^{n s} q^{(1 / 2) s(1-s)}\left(\begin{array}{c}
s-n-1 \\
s
\end{array}\right\rangle_{q}
$$


and

$$
\left\langle\begin{array}{l}
n \\
s
\end{array}\right\rangle_{1}=(-)^{s} q_{1}^{2 n s} q_{1}^{s(1-s)}\left(\begin{array}{c}
s-n-1 \\
s
\end{array}\right\rangle_{1}
$$

where

$$
\langle n\rangle_{1}=\frac{q_{1}^{2 n}-1}{q_{1}^{2}-1}, \quad\left\langle\begin{array}{l}
n \\
s
\end{array}\right\rangle_{1}=\left\langle\begin{array}{l}
n \\
s
\end{array}\right\rangle_{q_{1}^{2}} .
$$

Finally we note an alternative expression for the $q$-binomial coefficient, namely,

$$
\left\langle\begin{array}{l}
n \\
s
\end{array}\right\rangle_{q}=\frac{(q \mid q)_{n}}{(q \mid q)_{s}(q \mid q)_{n-s}}=\frac{\langle n\rangle !}{\langle s\rangle !\langle n-s\rangle !}
$$

where

$$
(a \mid q)_{n}=(1-a)(1-a q) \cdots\left(1-a q^{n-1}\right)
$$

is the $q$-shifted factorial function.

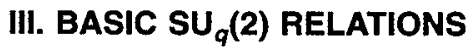
matrix

A generic element of the two-dimensional representation of $\mathrm{SU}_{q}(2)$ may be represented by the

$$
T=\left(\begin{array}{cc}
a & b \\
-q_{1} \bar{b} & \bar{a}
\end{array}\right)
$$

where

$$
a b=q b a, \quad a \bar{a}+b \bar{b}=1, \quad a \bar{b}=q \bar{b} a, \quad \bar{a} a+q_{1}^{2} \bar{b} b=1, \quad b \bar{b}=\bar{b} b .
$$

Let

$$
f_{n}=a^{n} \bar{a}^{n}
$$

Then

$$
f_{n}=a^{n-1}(a \bar{a}) \bar{a}^{n-1}=a^{n-1}(1-b \bar{b}) \bar{a}^{n-1}=f_{n-1}\left(1-q^{2(n-1)} b \bar{b}\right)
$$

and

$$
f_{n}=\left(1-q^{2} b \bar{b}\right)\left(1-\left(q^{2}\right)^{2} b \bar{b}\right) \cdots\left(1-\left(q^{2}\right)^{n-1} b \bar{b}\right)
$$

or

$$
a^{n} \bar{a}^{n}=\prod_{s=0}^{n-1}\left(1-q^{2 s} b \bar{b}\right)
$$

This function is the $q$-shifted factorial 


$$
(x \mid q)_{n}=\prod_{0}^{n-1}\left(1-q^{s} x\right)
$$

That is,

$$
a^{n} \bar{a}^{n}=\left(b \bar{b} \mid q^{2}\right)_{n}
$$

and in the same way

$$
\bar{a}^{n} a^{n}=\left(q_{1}^{2} b \bar{b} \mid q_{1}^{2}\right)_{n} .
$$

It is useful to have an expansion of the $q$-shifted factorial in powers of $x$. Set

$$
(x \mid q)_{n}=\sum_{s=0}^{n} a_{n s} x^{s}
$$

We have

$$
(x \mid q)_{n+1}=\left(1-q^{n} x\right)(x \mid q)_{n} .
$$

By Eqs. (3.8) and (3.9)

$$
a_{n+1, s}=a_{n s}-a_{n, s-1} q^{n}, \quad a_{n+1,0}=a_{n 0}=1, \quad a_{n+1, n+1}=-q^{n} a_{n n} .
$$

Try the ansatz

$$
a_{n s}=q^{n s} f(s)\left(\begin{array}{l}
n \\
s
\end{array}\right\rangle_{q_{1}} .
$$

By Eqs. (3.10) and (3.11)

$$
q^{s} \frac{\left\langle\begin{array}{c}
n+1 \\
s
\end{array}\right\rangle_{q_{1}}}{\left\langle\begin{array}{l}
n \\
s
\end{array}\right\rangle_{q_{1}}}=1-\frac{f(s-1)}{f(s)} \frac{\left\langle\begin{array}{c}
n \\
s-1
\end{array}\right\rangle_{q_{1}}}{\left\langle\begin{array}{l}
n \\
s
\end{array}\right\rangle_{q_{1}}}
$$

or

$$
f(s-1) / f(s)=-q^{s} .
$$

The solution of this functional equation is

$$
f(s)=(-)^{s} q^{-(s / 2)(s+1)}
$$

Therefore

$$
a_{n s}=q^{n s}(-)^{s} q^{-(s / 2)(s+1)}\left\langle\begin{array}{l}
n \\
s
\end{array}\right\rangle_{q_{1}}
$$




$$
\begin{gathered}
(x \mid q)_{n}=\sum_{s=0}^{n}(-)^{s} q^{n s} q^{-(s / 2)(s+1)}\left\langle\begin{array}{l}
n \\
s
\end{array}\right\rangle_{q_{1}} x^{s}, \\
\left(b \bar{b} \mid q^{2}\right)_{n}=\sum_{s=0}^{n}(-)^{s} q^{2 n s} q^{-s(s+1)}\left\langle\begin{array}{l}
n \\
s
\end{array}\right\rangle_{1}(b \bar{b})^{s},
\end{gathered}
$$

using

$$
\langle\rangle_{1}=\langle\rangle_{q_{1}^{2}}
$$

It is also useful to be able to commute $a$ through $\left(b \bar{b} \mid q^{2}\right)_{n}$. One has

$$
a\left(b \bar{b} \mid q_{1}^{2}\right)_{n}=a(1-b \bar{b})\left(1-q^{2} b \bar{b}\right) \cdots .
$$

Passing $a$ through each factor successively one obtains

$$
a\left(b \bar{b} \mid q^{2}\right)_{n}=\prod_{a}^{n-1}\left(1-q^{2} q^{2 s} b \bar{b}\right) a
$$

or

$$
a\left(b \bar{b} \mid q^{2}\right)_{n}=\left(q^{2} b \bar{b} \mid q^{2}\right)_{n} a
$$

Likewise

$$
\bar{a}\left(b \bar{b} \mid q^{2}\right)_{n}=\left(q_{1}^{2} b \bar{b} \mid q^{2}\right)_{n} \bar{a} .
$$

Higher order products may be reduced in a similar way. Thus

$$
a^{k_{1}} \bar{a}^{\ell_{1}} a^{k_{2}} \bar{a}^{\ell_{2}}=\left(a^{k_{1}} \bar{a}^{k_{1}}\right) \bar{a}^{\ell_{1}-k_{1}} a^{k_{2}-\ell_{2}}\left(a^{\ell} \bar{a}^{\ell}{ }_{2}\right)=\left(b \bar{b} \mid q^{2}\right)_{k_{1}}\left(\bar{a}^{\ell_{1}-k_{1}} a^{k_{2}-\ell_{2}}\right)\left(b \bar{b} \mid q^{2}\right) \ell_{2} .
$$

If, as we shall assume

$$
k_{1}+k_{2}=\ell_{1}+\ell_{2}
$$

then

$$
a^{k_{1}} \bar{a}^{\ell_{1}} a^{k_{2}} \bar{a}^{\ell}=\left(b \bar{b} \mid q^{2}\right)_{k_{1}}\left(q_{1}^{2} b \bar{b} \mid q_{1}^{2}\right)_{\ell_{1}-k_{1}}\left(b \bar{b} \mid q^{2}\right)_{\ell_{2}} .
$$

Alternative reductions are permitted by the algebra. For example,

$$
\begin{aligned}
a^{k_{1}} \bar{a}^{\ell} a^{k_{2}} \bar{a}^{\ell} \ell_{2} & =\left(a^{k_{1}} \bar{a}^{k_{1}}\right) \bar{a}^{\ell_{1}-k_{1}}\left(a^{k_{2}} \bar{a}^{k_{2}}\right) \bar{a}^{\ell} \ell_{2}-k_{2}=\left(b \bar{b} \mid q^{2}\right)_{k_{1}} \bar{a}^{\ell_{1}-k_{1}}\left(b \bar{b} \mid q^{2}\right)_{k_{2}} \bar{a}^{\ell_{2}-k_{2}} \\
& =\left(b \bar{b} \mid q^{2}\right)_{k_{1}}\left(q_{1}^{2\left(\ell_{1}-k_{1}\right)} b \bar{b} \mid q^{2}\right)_{k_{2}} \bar{a}^{\ell_{1}-k_{1}} \bar{a}^{\ell}-k_{2} .
\end{aligned}
$$

Again under the assumption (3.24)

$$
a^{k_{1}} \bar{a}^{\ell} a^{k_{2}} \bar{a}^{\ell_{2}}=\left(b \bar{b} \mid q^{2}\right)_{k_{1}}\left(q_{1}^{2\left(\ell_{1}-k_{1}\right)} b \bar{b} \mid q^{2}\right)_{k_{2}} .
$$

By Eqs. (3.25) and (3.27) 


$$
\left(q_{1}^{2} b \bar{b} \mid q_{1}^{2}\right)_{\ell_{1}-k_{1}}\left(b \bar{b} \mid q^{2}\right)_{\ell_{2}}=\left(q_{1}^{2\left(\ell_{1}-k_{1}\right)} b \bar{b} \mid q^{2}\right)_{k_{2}}, \quad \ell_{1}-k_{1}=k_{2}-\ell_{2}
$$

or

$$
\left(q_{1}^{2} b \bar{b} \mid q_{1}^{2}\right)_{n}\left(b \bar{b} \mid q^{2}\right)_{m}=\left(q_{1}^{2 n} b \bar{b} \mid q^{2}\right)_{n+m}
$$

Finally

$$
\begin{aligned}
& a^{k_{1}} \bar{a}^{\ell_{1}} a^{k_{2}} \bar{a}^{\ell}{ }_{2}=a^{k_{1}}\left(\bar{a}^{\ell}{ }_{1} a^{\ell_{1}}\right) a^{k_{2}-\ell_{1}} \bar{a}^{\ell_{2}}=a^{k_{1}}\left(q_{1}^{2} b \bar{b} \mid q_{1}^{2}\right) \ell_{1} a^{k_{2}-\ell_{1}} \bar{a}^{\ell} \\
& =\left(q^{2 k_{1}} q_{1}^{2} b \bar{b} \mid q_{1}^{2}\right)_{\ell_{1}} a^{k_{1}+k_{2}-\ell_{1}} \bar{a}_{2} \\
& =\left(q^{2 k_{1}} q_{1}^{2} b \bar{b} \mid q_{1}^{2}\right) \ell_{1}\left(b \vec{b} \mid q^{2}\right) \ell_{2} .
\end{aligned}
$$

By Eqs. (3.25) and (3.30)

$$
\left(b \bar{b} \mid q^{2}\right)_{k_{1}}\left(q_{1}^{2} b \bar{b} \mid q_{1}^{2}\right)_{\ell_{1}-k_{1}}=\left(q^{2 k_{1}} q_{1}^{2} b \bar{b} \mid q_{1}^{2}\right)_{\ell_{1}}
$$

or

$$
\left(b \bar{b} \mid q^{2}\right)_{n}\left(q_{1}^{2} b \bar{b} \mid q_{1}^{2}\right)_{m}=\left(q^{2 n-2} b \bar{b} \mid q^{2}\right)_{n+m}
$$

and also by Eqs. (3.29) and (3.31a)

$$
\left(q_{1}^{2 n} b \bar{b} \mid q^{2}\right)_{n+m}=\left(q^{2 m-2} b \bar{b} \mid q_{1}^{2}\right)_{n+m} .
$$

By Eqs. (3.30) and (3.32) we have

$$
a^{k_{1}} \bar{a}^{\ell_{1}} a^{k_{2}} \bar{a}^{\ell_{2}}=\left(q^{2\left(k_{1}-\ell_{1}\right)} b \bar{b} \mid q^{2}\right)_{\ell_{1}}\left(b \bar{b} \mid q^{2}\right) \ell_{2} .
$$

These special relations are properties of the shifted factorial function $\left(x \mid q^{2}\right)_{n}$.

\section{THE ORTHOGONALITY RELATIONS AND RELATED IDENTITIES}

We shall make essential use of a linear operator, $h$, that plays the role of the Haar measurc in the Woronowicz theory. ${ }^{5}$ Here it is simply a linear operator with the following additional property:

$$
h\left(a^{s} b^{n} \bar{b}^{m}\right)=\delta^{s 0} \delta^{n m} q^{m} /[m+1]_{q},
$$

where

$$
[n]_{q}=\frac{q^{n}-q^{-n}}{q-q^{-1}} .
$$

We shall refer to $h$ as the Haar operator. Woronowicz has obtained the following orthogonality relations:

$$
h\left(D_{m \mu}^{j} \bar{D}_{m^{\prime} \mu^{\prime}}^{j^{\prime}}\right)=\delta^{j j^{\prime}} \delta_{m m^{\prime}} \delta_{\mu \mu^{\prime}} q^{-2 \mu /[2 j+1]_{q}}
$$

and he has also introduced a formal integration

$$
\int_{W} f(\alpha) \omega_{0} \wedge \omega_{1} \wedge \omega_{2}=h(f(\alpha))
$$


where $f(\alpha)$ lies in the algebra generated by $(a, \bar{a}, b, \bar{b})$. Here $\omega_{0}, \omega_{1}, \omega_{2}$ are independent one-forms on the algebra and the exterior product $\omega_{0} \wedge \omega_{1} \wedge \omega_{2}$ may be interpreted as a formal invariant volume element which we may denoted by $d \tau$. Then

$$
\int_{W} D_{m \mu}^{j} \bar{D}_{m^{\prime} \mu^{\prime}}^{j^{\prime}} d \tau=\delta^{j j^{\prime}} \delta_{m m^{\prime}} \delta_{\mu \mu} q^{-2 \mu} /[2 j+1]_{q}
$$

and

$$
\int_{W} d \tau=h(1)=1
$$

One may compare Eq. (4.3b) with the usual orthogonality relations for the Wigner functions

$$
\int D_{m \mu}^{j} \bar{D}_{m^{\prime} \mu^{\prime}}^{j^{\prime}} d \tau=\delta_{m m^{\prime}} \delta_{\mu \mu^{\prime}} \delta^{j^{\prime}}(2 j+1)^{-1} \int d \tau
$$

The relations (4.3) are the corresponding orthogonality relations of the $q$-Wigner functions, special cases of which are the $q$-Jacobi polynomials and the $q$-spherical harmonics.

The irreducible representations $D_{m n}^{j}$ have been obtained by several authors. ${ }^{7}$ We have obtained them in the following form: ${ }^{6}$

$$
D_{m \ell}^{j}=\Delta_{m \ell}^{j} \sum\left\langle\begin{array}{c}
n_{+} \\
s
\end{array}\right\rangle_{1}\left\langle\begin{array}{c}
n_{-} \\
t
\end{array}\right\rangle_{1} q_{1}^{\left(n_{+}-s+1\right) t}(-)^{t} \delta\left(s+t, n_{+}^{\prime}\right) a^{s} b^{n_{+}-s} \bar{b}^{t} \bar{a}^{n_{-}-t},
$$

where

$$
\Delta_{m \ell}^{j}=\left(\frac{\langle j+\ell\rangle_{1} !\langle j-\ell\rangle_{1} !}{\langle j+m\rangle_{1} !\langle j-m\rangle_{1} !}\right)^{1 / 2}, \quad n_{ \pm}=j \pm m, \quad n_{ \pm}^{\prime}=j \pm \ell
$$

When substituted into Eq. (4.3) one obtains identities among the $q$-shifted binomial coefficients. In examining these identities we shall record just the case in which the right side does not vanish.

The simplest of these identities may be obtained as follows:

$$
h\left(D_{j j}^{j} \bar{D}_{j j}^{j}\right)=q^{-2 j} /[2 j+1]_{q} .
$$

By Eq. (4.5) the left side of the preceding equation may be reduced. Then

$$
h\left(a^{2 j} \bar{a}^{2 j}\right)=q^{-2 j} /[2 j+1]_{q}
$$

or by Eq. (3.6)

$$
h\left(\left(b \bar{b} \mid q^{2}\right)_{2 j}\right)=q^{-2 j} /[2 j+1]_{q} .
$$

On the other hand, by Eqs. (3.17) and (4.1) one has the identity

$$
\sum_{s=0}^{2 j}(-)^{s} q^{4 j s} q^{-s(s+1)}\left\langle\begin{array}{c}
2 j \\
s
\end{array}\right\rangle_{1} \frac{q^{s}}{[s+1]_{q}}=\frac{q^{-2 j}}{[2 j+1]_{q}}
$$

In the limit $q \rightarrow 1$, Eq. (4.7) becomes 


$$
\sum_{s=0}^{n}(-)^{s}\left(\begin{array}{l}
n \\
s
\end{array}\right) \frac{1}{s+1}=\frac{1}{n+1}
$$

where $n=2 j$. The next simplest case is

$$
h\left(D_{j \lambda}^{j} \bar{D}_{j \lambda}^{j}\right)=q^{-2 \lambda} /[2 j+1]_{q} .
$$

This equation reduces to

$$
h\left((b \bar{b})^{m}\left(b \bar{b} \mid q^{2}\right)_{n}\right)=B_{q}(m+1, n+1),
$$

where

$$
B_{q}(m+1, n+1)=\frac{\langle m\rangle_{1} !\langle n\rangle_{1} !}{\langle m+n\rangle_{1} !} \frac{q^{m-n-2 m n}}{[m+n+1]_{q}} .
$$

Here we have set

$$
m=j-\lambda, \quad n=j+\lambda
$$

We shall refer to $B_{q}(m+1, n+1)$ as a $q$-beta function. There are these special cases of Eq. (4.10)

$$
\begin{gathered}
h\left((b \bar{b})^{m}\right)=\frac{q^{n}}{[m+1]_{q}}, \\
h\left(\left(b \bar{b} \mid q^{2}\right)_{n}\right)=\frac{q^{-n}}{[n+1]_{q}} .
\end{gathered}
$$

Again substituting from Eq. (3.17) into Eq. (4.10) one gets

$$
\sum_{s=0}^{n}(-)^{s} q^{2 n s} q^{-s(s+1)}\left\langle\begin{array}{l}
n \\
s
\end{array}\right\rangle_{1} \frac{q^{m+s}}{[m+s+1]_{q}}=B_{q}(m+1, n+1)
$$

When $q=1$, Eq. (4.14) becomes

$$
\sum_{s=0}^{n}(-)^{s}\left(\begin{array}{l}
n \\
s
\end{array}\right) \frac{1}{m+s+1}=B(m+1, n+1) .
$$

We may now utilize Eqs. (3.17) and (4.10) to calculate

$$
h\left((b \bar{b})^{m}\left(x b \bar{b} \mid q^{2}\right)_{p}\left(b \bar{b} \mid q^{2}\right)_{n}\right)
$$

in the following way. Multiply Eq. (4.10) by $\left(x b \bar{b} \mid q^{2}\right)_{p}$ as given in Eq. (3.17)

$$
\begin{aligned}
\sum_{0}^{p}(-x)^{s} q^{2 p s-s(s+1)}\left\langle\begin{array}{c}
p \\
s
\end{array}\right\rangle_{q_{1}^{2}} h\left((b \bar{b})^{m+s}\left(b \bar{b} \mid q^{2}\right)_{n}\right)= & \sum(-x)^{s} q^{2 p s-s(s+1)}\left\langle\begin{array}{l}
p \\
s
\end{array}\right\rangle_{q_{1}^{2}} \\
& \times B_{q}(m+s+1, n+1)
\end{aligned}
$$

or 


$$
h\left((b \bar{b})^{m}\left(x b \bar{b} \mid q^{2}\right)_{p}\left(b \bar{b} \mid q^{2}\right)_{n}\right)=\sum(-)^{s} q^{2 p s-s(s+1)}\left\langle\begin{array}{c}
p \\
s
\end{array}\right\rangle_{1} x^{s} B_{q}(m+s+1, n+1)
$$

With this result let us now consider the most general expansion of the orthogonality relations

$$
\begin{aligned}
& h\left(D_{\mu \lambda}^{j} \bar{D}_{\mu^{\prime} \lambda^{\prime}}^{j^{\prime}}\right)=\delta^{j j^{\prime}} \delta_{\mu \mu^{\prime}} \delta_{\lambda \lambda^{\prime}}\left(\Delta_{\mu \lambda}^{j}\right)^{2} \sum\left\langle\begin{array}{c}
n_{+} \\
s_{1}
\end{array}\right\rangle_{1}\left\langle\begin{array}{c}
n_{-} \\
t_{1}
\end{array}\right\rangle_{1} q_{1}^{\left(n_{+}-s_{1}+1\right) t_{1}}(-)^{t_{1}} \\
& \times \sum\left\langle\begin{array}{c}
n_{+} \\
s_{2}
\end{array}\right\rangle_{1}\left\langle\begin{array}{c}
n_{-} \\
t_{2}
\end{array}\right\rangle_{1} q_{1}^{\left(n_{+}-s_{2}+1\right) t_{2}}(--)^{t_{2}} \delta\left(s_{1}+t_{1}, n_{+}^{\prime}\right) \delta\left(s_{2}+t_{2}, n_{+}^{\prime}\right) \\
& \times h\left(\left(a^{s_{1}} b^{n_{+}-s_{1}} \bar{b}^{t_{1}} \bar{a}^{n_{-}-t_{1}}\right)\left(a^{n_{-}-t_{2}} b^{t_{2}} \bar{b}^{n_{+}-s_{2}} \bar{a}^{s_{2}}\right)\right),
\end{aligned}
$$

where

$$
n_{ \pm}=j \pm \mu, \quad n_{ \pm}^{\prime}=j \pm \lambda
$$

Because of the $\delta$ functions in Eq. (4.18) there are only two independent sums on the right-hand side. In addition, by Eq. (4.1)

$$
s_{1}+t_{1}=s_{2}+t_{2}
$$

The $h$ "integral" may be evaluated by first separating the $a$ and $b$ products

$$
I=h\left(a^{s_{1}} \cdots \bar{a}^{n_{-}-t_{1}} a^{n_{-}-t_{2}} \cdots \bar{a}^{s_{2}}\right)=h\left(a^{s_{1}} \bar{a}^{n_{-}-t_{1}} a^{n_{-}-t_{2}} \bar{a}^{s_{2}}\right)(b \bar{b})^{n_{+}+t_{2}-s_{1}} q^{\theta}
$$

where

$$
\theta=\left(n_{+}+t_{1}-s_{1}\right) s_{1}+\left(n_{+}+t_{2}-s_{2}\right) s_{2}
$$

and we have used Eq. (4.20).

The $a$ product may now be simplified by Eq. (3.27)

$$
a^{s_{1}} \tilde{a}^{n_{-}-t_{1}} a^{n_{-}-t_{2}} \bar{a}^{s_{2}}=\left(b \vec{b} \mid q^{2}\right)_{s_{1}}\left(q_{1}^{2\left(n_{-}-t_{1}-s_{1}\right)} b \bar{b} \mid q^{2}\right)_{n_{-}-t_{2}}
$$

Then

$$
I=q^{\theta} h\left((b \bar{b})^{n_{+}+t_{2}-s_{1}}\left(x b \bar{b} \mid q^{2}\right)_{n_{-}-t_{2}}\left(b \bar{b} \mid q^{2}\right)_{s_{1}}\right),
$$

where

$$
x=q_{1}^{2\left(n_{-}-t_{1}-s_{1}\right)}=q^{2(\mu+\lambda)} .
$$

By Eq. (4.17)

$$
I=q^{\theta} \sum_{\ell}(-)^{\ell} q^{2\left(n_{-}-t_{2}\right) \ell-\ell(\ell+1)}\left\langle\begin{array}{c}
n_{-}-t_{2} \\
\ell^{\prime}
\end{array}\right\rangle_{1} x^{\ell} B_{q}\left(n_{-}-t_{2}+\ell+1, s_{1}+1\right)
$$

and by Eq. (4.18) 


$$
\begin{aligned}
h\left(D_{\mu \lambda}^{j} \bar{D}_{\mu^{\prime} \lambda^{\prime}}^{j^{\prime}}\right)= & \delta^{j j^{\prime}} \delta_{\mu \mu^{\prime}} \delta_{\lambda \lambda^{\prime}}\left(\Delta_{\mu \lambda}^{j}\right)^{2} \sum\left\langle\begin{array}{c}
n_{+} \\
s_{1}
\end{array}\right)_{1}\left\langle\begin{array}{c}
n_{-} \\
t_{1}
\end{array}\right\rangle_{1}\left\langle\begin{array}{c}
n_{+} \\
s_{2}
\end{array}\right)_{1}\left\langle\begin{array}{c}
n_{-} \\
t_{2}
\end{array}\right\rangle_{1} \delta\left(s_{1}+t_{1}, n_{+}^{\prime}\right) \delta\left(s_{2}\right. \\
& \left.+t_{2}, n_{+}^{\prime}\right) q_{1}^{\left(n_{+}+1\right)\left(t_{1}+t_{2}\right)-s_{1} t_{1}-s_{2} t_{2}}(-)^{t_{1}+t_{2}} q^{\theta} \sum_{\ell}(-)^{\ell} q^{2\left(n_{-}-t_{2}\right) \ell-\ell(\ell+1)} \\
& \times\left(\begin{array}{c}
n_{-}-t_{2} \\
\ell
\end{array} q_{1} q^{2(\mu+\lambda) \ell_{B}\left(n_{-}-t_{2}+\ell+1, s_{1}+1\right) .}\right.
\end{aligned}
$$

In the case that $h\left(D_{\mu \lambda}^{j} \bar{D}_{\mu^{\prime} \lambda^{\prime}}^{j^{\prime}}\right)$ does not vanish, one has finally

$$
\begin{aligned}
& \left\langle\begin{array}{c}
2 j \\
j-\lambda
\end{array}\right\rangle_{1}^{-1} \sum_{t_{1} t_{2}}\left\langle\begin{array}{c}
n_{+} \\
s_{1}
\end{array}\right\rangle_{1}\left\langle\begin{array}{c}
n_{-} \\
t_{1}
\end{array}\right\rangle_{1}\left\langle\begin{array}{c}
n_{+} \\
s_{2}
\end{array}\right\rangle_{1}\left\langle\begin{array}{c}
n_{-} \\
t_{2}
\end{array}\right\rangle_{1} \delta\left(s_{1}+t_{1}, n_{+}^{\prime}\right) \delta\left(s_{2}+t_{2}, n_{+}^{\prime}\right)(-)^{t_{1}+t_{2}} q^{\varphi} \\
& \quad \times \sum_{\ell}(-)^{\prime} q^{2(\mu+\lambda) \ell} q^{\psi}\left(\begin{array}{c}
n_{-}-t_{2} \\
\ell
\end{array}\right\rangle_{1} B_{q}\left(n_{-}-t_{2}+\ell+1, s_{1}+1\right)=q^{2 n_{+} n_{-}} B_{q}\left(n_{+}+1, n_{-}+1\right),
\end{aligned}
$$

where

$$
\begin{gathered}
\varphi=\left(n_{+}+1\right)\left(t_{1}+t_{2}\right)-s_{1} t_{1}-s_{2} t_{2}-s_{1}\left(s_{1}-t_{1}-n_{+}\right)+s_{2}\left(s_{2}-t_{2}-n_{+}\right), \\
\psi=\ell(\ell+1)-2\left(n_{-}-t_{2}\right) \ell .
\end{gathered}
$$

\section{THE CLEBSCH-GORDAN COEFFICIENTS}

In order to decompose a direct product representation into a sum of irreducible parts we write

$$
\begin{aligned}
D_{\mu \lambda}^{j} D_{\mu^{\prime} \lambda^{\prime}}^{j^{\prime}} & =\sum_{J^{\prime} M^{\prime} \Lambda^{\prime}}\left\langle\mu \mu^{\prime}\left|\left(S^{J^{\prime}}\right)^{+}\right| M^{\prime}\right\rangle\left\langle M^{\prime}\left|D^{J^{\prime}}\right| \Lambda^{\prime}\right\rangle\left\langle\Lambda^{\prime}\left|S^{J^{\prime}}\right| \lambda \lambda^{\prime}\right\rangle \\
& =\sum_{J^{\prime} M^{\prime} \Lambda^{\prime}}\left\langle M^{\prime}\left|S^{J^{\prime}}\right| \mu \mu^{\prime}\right\rangle^{*}\left\langle\Lambda^{\prime}\left|S^{J^{\prime}}\right| \lambda \lambda^{\prime}\right\rangle\left\langle M^{\prime}\left|D^{J^{\prime}}\right| \Lambda^{\prime}\right\rangle .
\end{aligned}
$$

Then

$$
\begin{aligned}
h\left(D_{\mu \lambda}^{j} D_{\mu^{\prime} \lambda^{\prime}}^{j^{\prime}} \bar{D}_{M \Lambda}^{J}\right) & =\sum\left\langle M^{\prime}\left|S^{J^{\prime}}\right| \mu \mu^{\prime}\right\rangle^{*}\left\langle\Lambda^{\prime}\left|S^{J^{\prime}}\right| \lambda \lambda^{\prime}\right\rangle h\left(D_{M^{\prime} \Lambda^{\prime}}^{J^{\prime}} \bar{D}_{M \Lambda}^{J}\right) \\
& =\left\langle M\left|S^{J}\right| \mu \mu^{\prime}\right\rangle^{*}\left\langle\Lambda\left|S^{J}\right| \lambda \lambda^{\prime}\right\rangle q_{1}^{2 \Lambda} /[2 J+1]_{q}
\end{aligned}
$$

or

$$
h\left(D_{\mu \lambda}^{j} D_{\mu^{\prime} \lambda^{\prime}}^{j^{\prime}} \bar{D}_{M \Lambda}^{J}\right)=\left\langle M\left|S^{J}\right| \mu \mu^{\prime}\right\rangle\left\langle\Lambda\left|S^{J}\right| \lambda \lambda^{\prime}\right\rangle q_{1}^{2 \Lambda} /[2 J+1]_{q}
$$

If we take all the matrix elements of $S^{J}$ to be real in Eq. $(5.3 \mathrm{~b})$, we shall use this equation in two ways: first to determine the Clebsch-Gordan coefficients (the elements of the matrix $S^{J}$ ) and second to obtain a general expression for the Woronowicz integral of the triple product

$$
\int_{W} D_{\mu \lambda}^{j} D_{\mu^{\prime} \lambda^{\prime}}^{j^{\prime}} \bar{D}_{M \Lambda}^{J} d \tau
$$


in the notation of Eq. (4.3a). In the SU(2) case one has instead of Eq. (5.4) the Hurwitz integral over the group space. In both cases the integral vanishes unless angular momentum is conserved in the quantum sense: ${ }^{7}$

$$
J=j+j^{\prime}, \ldots,\left(j-j^{\prime}\right)
$$

and the $z$ component of the angular momentum is conserved

$$
M=\mu+\mu^{\prime}, \quad \Lambda=\lambda+\lambda^{\prime} .
$$

We examine some special cases. Let us first consider $J=j+j^{\prime}$. We have

$$
h\left(D_{j j}^{j} D_{j^{\prime} j^{\prime}}^{j^{\prime}} \bar{D}_{J J}^{J}\right)=\left(S_{j j^{\prime}}^{J\left(j j^{\prime}\right)}\right)^{*}\left(S_{j j^{\prime}}^{J\left(j j^{\prime}\right)}\right)_{j j^{\prime}} q_{1}^{2 J} /[2 J+1]_{q} .
$$

But

$$
D_{j j}^{j}=a^{2 j}
$$

Then

$$
\begin{aligned}
\left|S_{j j^{\prime}}^{J\left(j j^{\prime}\right)}\right|^{2} & =q^{2 J}[2 J+1]_{q} h\left(a^{2 j} a^{2 j^{\prime}} \bar{a}^{2 J}\right)=q^{2 J}[2 J+1]_{q} h\left(a^{2 J} \bar{a}^{2 J}\right), \quad \text { if } \quad j+j^{\prime}=J \\
& =q^{2 J}[2 J+1]_{q} q^{-2 J}[2 J+1]_{q}^{-1}
\end{aligned}
$$

by Eq. $(4.6 \mathrm{~b})$ or

$$
\left|S_{j j^{\prime}}^{J\left(j j^{\prime}\right)}\right|=1
$$

and

$$
S_{j j^{\prime}}^{J\left(j j^{\prime}\right)}=1
$$

since these elements are all real and positive. Then by Eq. (5.3)

$$
S_{\lambda \lambda^{\prime}}^{J\left(j j^{\prime}\right)}=\left(q_{1}^{2}\right)^{-\Lambda}[2 J+1]_{q} h\left(D_{j \lambda}^{j} D_{j^{\prime} \lambda^{\prime}}^{j^{\prime}} \bar{D}_{j+j^{\prime}, \lambda+\lambda^{\prime}}^{J}\right), \quad J=j+j^{\prime}
$$

or

$$
\begin{aligned}
S_{\lambda \lambda^{\prime}}^{J\left(j j^{\prime}\right)}= & q^{2 \Lambda}[2 J+1]_{q} \Delta_{j j}^{j} \Delta_{j^{\prime} j^{\prime}}^{j^{\prime}} \Delta_{J \Lambda}^{J}\left\langle\begin{array}{c}
2 j \\
j+\lambda
\end{array}\right\rangle_{1}\left\langle\begin{array}{c}
2 j^{\prime} \\
j^{\prime}+\lambda^{\prime}
\end{array}\right\rangle_{1}\left\langle\begin{array}{c}
2 J \\
J+\Lambda
\end{array}\right\rangle_{1} \\
& \times h\left(a^{j+\lambda} b^{j-\lambda} a^{j^{\prime}+\lambda^{\prime}} b^{j^{\prime}-\lambda^{\prime}} \bar{b}^{J-\Lambda} \bar{a}^{J+\Lambda}\right) .
\end{aligned}
$$

One finds

$$
h\left(a^{j+\lambda} b^{j-\lambda} a^{j^{\prime}+\lambda^{\prime}} b^{j^{\prime}-\lambda^{\prime}} \bar{b}^{J-\Lambda} \tilde{a}^{J+\Lambda}\right)=q_{1}^{2(J-\Lambda)(J+\Lambda)+(j-\lambda)\left(j^{\prime}+\lambda^{\prime}\right)} h\left((b \bar{b})^{J-\Lambda}\left(b \bar{b} \mid q^{2}\right)_{J+\Lambda}\right)
$$

The expression (5.10) may be evaluated by Eq. (4.10). Collecting these results one finds

$$
S_{\lambda \lambda^{\prime}}^{J\left(j j^{\prime}\right)}=q_{1}^{\sigma}\left(\begin{array}{c}
2 j \\
j+\lambda
\end{array}\right\rangle_{1}^{1 / 2}\left(\begin{array}{c}
2 j^{\prime} \\
j^{\prime}+\lambda^{\prime}
\end{array}\right\rangle_{1}^{1 / 2}\left\langle\begin{array}{c}
2 J \\
J+\Lambda
\end{array}\right\rangle_{1}^{-1 / 2}, \text { if } J=j+j^{\prime},
$$

where 


$$
\sigma=(j-\lambda)\left(j^{\prime}+\lambda^{\prime}\right)
$$

By a similar argument one finds if $J=j-j^{\prime}$

$$
\begin{gathered}
S_{\lambda \lambda^{\prime}}^{J\left(j j^{\prime}\right)}=q_{1}^{\tau}\left\{\frac{\langle 2 J+1\rangle_{1}}{\langle 2 j+1\rangle_{1}}\left(\begin{array}{c}
2 j^{\prime} \\
j^{\prime}+\lambda^{\prime}
\end{array}\right\rangle_{1}^{-1}\left(\begin{array}{c}
2 j \\
j+\lambda
\end{array}\right)_{1}^{-1}\left(\begin{array}{c}
2 J \\
J+\Lambda
\end{array}\right)_{1}\right\}^{1 / 2}(-)^{j^{\prime}+\lambda^{\prime}}, \\
\tau=j^{\prime}-\lambda^{\prime}+\left(j^{\prime}-\lambda^{\prime}\right)(J-\Lambda),
\end{gathered}
$$

where

$$
\Lambda=\lambda+\lambda^{\prime}
$$

\section{GENERAL CASE (PART A)}

To obtain $S_{\lambda \lambda^{\prime}}^{J\left(j j^{\prime}\right)}$ for an unrestricted value of $J$ we follow the method of Wigner for the $q=1$ case by first considering the integral

$$
h\left(D_{j j}^{j} D_{-j^{\prime}-j^{\prime}}^{j} \bar{D}_{\Delta \Delta}^{J}\right)=\left|S_{j-j^{\prime}}^{J}\right|^{2} q_{1}^{2 \Delta} /[2 J+1]_{q}, \quad \Delta=j-j^{\prime} .
$$

By Eq. (4.5)

$$
\begin{gathered}
D_{j j}^{j}=a^{2 j}, \quad D_{-j^{\prime}-j^{\prime}}^{j^{\prime}}=\bar{a}^{2 j^{\prime}}, \\
\bar{D}_{\Delta \Delta}^{J}=\sum\left\langle\begin{array}{c}
N_{+} \\
T
\end{array}\right\rangle_{1}\left(\begin{array}{c}
N_{-} \\
T
\end{array}\right\rangle_{1} q_{1}^{T(T+1)}(-)^{T}\left(a^{N_{-}-T} b^{T} \bar{b}^{N_{+}-S} \bar{a}^{S}\right) \delta\left(S+T, N_{+}\right) .
\end{gathered}
$$

One then finds by Eqs. (3.2) and (3.33)

$$
\begin{aligned}
h\left(D_{j j}^{j} D_{-j^{\prime}-j^{\prime}}^{j^{\prime}} \bar{D}_{\Delta \Delta}^{J}\right)= & \sum_{T}\left\langle\begin{array}{c}
N_{+} \\
T
\end{array}\right\rangle_{1}\left\langle\begin{array}{c}
N_{-} \\
T
\end{array}\right\rangle_{1} q_{1}^{T(T+1)}(-)^{T} q^{2 T S} \delta\left(T+S, N_{+}\right) \\
& \times h\left((b \bar{b})^{T}\left(b \bar{b} \mid q^{2}\right)_{S}\left(q^{4 \Delta} b \bar{b} \mid q^{2}\right)_{2 j^{\prime}}\right) .
\end{aligned}
$$

The $h$ factor in Eq. (6.3) is of the same form as the $h$ factor in Eq. (4.15) and can be represented as a power series by the same procedure used to obtain Eq. (4.17). One then finds

$$
h\left((b \bar{b})^{T}\left(b \bar{b} \mid q^{2}\right)_{s}\left(q^{4 \Delta} b \bar{b} \mid q^{2}\right)_{2 j^{\prime}}\right)=\sum_{\ell}(-)^{\ell} q^{2 m \ell} q^{-\ell^{2}-\ell}\left|\begin{array}{c}
m \\
\ell
\end{array}\right\rangle_{1} q^{4 \Delta \ell} B_{q}(T+\ell+1, S+1),
$$

where

$$
m=2 j^{\prime} .
$$

After interchanging the order of summation we rewrite Eq. (6.3)

$$
\begin{aligned}
h\left(D_{j j}^{j} D_{-j^{\prime}-j^{\prime}}^{j^{\prime}} \bar{D}_{\Delta \Delta}^{J}\right)= & \sum_{\ell}(-)^{\ell} q^{4 j \ell} q^{-\ell^{2}-\ell}\left\langle\begin{array}{c}
m \\
\ell
\end{array}\right)_{1} \sum_{T}\left\langle\begin{array}{c}
N_{+} \\
T
\end{array}\right\rangle_{1}\left\langle\begin{array}{c}
N_{-} \\
T
\end{array}\right\rangle_{1} q^{2 T S-T(T+1)} \\
& \times(-)^{T} \delta\left(T+S, N_{+}\right) B_{q}(T+\ell+1, S+1) .
\end{aligned}
$$

Denote the $S, T$-sum by $B$. Then 


$$
\begin{gathered}
B=\sum_{S, T}(-)^{T}\left(\begin{array}{c}
N_{+} \\
T
\end{array}\right\rangle_{1}\left\langle\begin{array}{c}
N_{-} \\
T
\end{array}\right\rangle_{1} q^{2 r S-T(T+1)} \delta\left(T+S, N_{+}\right) \frac{\langle S\rangle_{1} !\langle T+\ell\rangle_{1} !}{\langle S+T+\ell\rangle_{1} !} \frac{q^{T+\ell-S-2 S(T+\ell)}}{[S+T+\ell+1]_{q}} \\
B=\frac{q^{-N_{+}} q^{\left(1-2 N_{+}\right) \ell}}{\left[N_{+}+\ell+1\right]_{q}} \frac{\left\langle N_{+}\right\rangle_{1} !\langle\ell\rangle_{1} !}{\left\langle N_{+}+\ell\right\rangle_{1} !} \sum_{T}(-)^{T}\left(\begin{array}{c}
T+\ell \\
T
\end{array}\right\rangle_{1}\left\langle\begin{array}{c}
N_{-} \\
T
\end{array}\right\rangle_{1} q^{-T^{2}+T} q^{2 T \ell}
\end{gathered}
$$

By Eq. (2.9)

$$
\left\langle\begin{array}{c}
T+\mathscr{C} \\
T
\end{array}\right\rangle_{1}=(-)^{T} q_{1}^{2 T(T+\cap)} q_{1}^{T(1-T)}\left(\begin{array}{c}
-\ell-1 \\
T
\end{array}\right\rangle_{1}
$$

Then the summation in Eq. (6.7) becomes

$$
\sum_{T}=\sum_{T}(-)^{2 T}\left(\begin{array}{c}
N_{-} \\
T
\end{array}\right)_{1}\left(\begin{array}{c}
-\ell-1 \\
T
\end{array}\right)_{1} q^{-2 T^{2}}
$$

Set $t=N_{-}-T$

$$
\sum_{T}=\sum_{t}\left\langle\begin{array}{c}
N_{-} \\
t
\end{array}\right\rangle_{1}\left\langle\begin{array}{c}
-\ell-1 \\
N_{-}-t
\end{array}\right\rangle_{1} q^{-2\left(N_{-}-t\right)^{2}}=\left(\begin{array}{c}
N_{-}-\ell-1 \\
N_{-}
\end{array}\right\rangle_{1}
$$

and

$$
B=\frac{q^{-N_{+}} q^{\left(1-2 N_{+}\right)}}{\left[N_{+}+\ell+1\right]_{q}} \frac{\left\langle N_{+}\right\rangle_{1} !\langle\ell\rangle_{1} !}{\left\langle N_{+}+\ell\right\rangle_{1} !}\left(\begin{array}{c}
N_{-}-\ell-1 \\
N_{-}
\end{array}\right\rangle_{1}
$$

Before substituting into the original expression convert all brackets to the form \langle\rangle$_{1}$. Then

$$
\frac{[2 J+1]_{q}}{\left[N_{+}+\ell+1\right]_{q}}=q^{2 J-N_{+}-\ell} \frac{\langle 2 J+1\rangle_{1}}{\left\langle N_{+}+\ell+1\right\rangle_{1}} .
$$

Returning to Eq. (6.1), substituting from Eqs. (6.5) and (6.11), we get

$$
\begin{aligned}
\left|S_{j-j^{\prime}}^{J\left(j j^{\prime}\right)}\right|^{2}= & \langle 2 J+1\rangle_{1}\left\langle N_{+}\right\rangle_{1} !\left\langle 2 j^{\prime}\right\rangle_{1} ! \sum_{\ell}(-)^{\ell} q^{2\left(j+j^{\prime}-J\right) \ell} q^{-\ell^{2}-\ell} \\
& \times\left\langle\begin{array}{c}
N_{-}-\ell-1 \\
N_{-}
\end{array}\right\rangle_{1} \frac{1}{\left\langle 2 j^{\prime}-\ell\right\rangle_{1} !} \frac{1}{\left\langle N_{+}+\ell+1\right\rangle_{1} !}
\end{aligned}
$$

Let $f=-\lambda-1$. Then

$$
\left|S_{j-j^{\prime}}^{J\left(j j^{\prime}\right)}\right|^{2}=\langle 2 J+1\rangle_{1} \frac{\left\langle 2 j^{\prime}\right\rangle_{1} !\left\langle N_{-}-2 j^{\prime}-1\right\rangle_{1} !}{\left\langle N_{-}\right\rangle_{1} !} q^{2\left(J-j-j^{\prime}\right)} I
$$

where the $\lambda$ sum is given by

$$
I=\sum_{\lambda}(-)^{-\lambda-1} q^{2\left(J-j-j^{\prime}\right) \lambda} q^{-\lambda^{2}-\lambda}\left(\begin{array}{c}
N_{+} \\
N_{+}-\lambda
\end{array}\right)\left(\begin{array}{c}
N_{-}+\lambda \\
2 j^{\prime}+1+\lambda
\end{array}\right)_{1} .
$$


Now set $\lambda=N_{+}-\mu$

$$
I=q^{\sigma_{1}} \sum_{\mu}(-)^{-N_{+}-1}(-)^{\mu} q^{4 j \mu} q^{-\mu^{2}+\mu}\left\langle\begin{array}{c}
N_{+} \\
\mu
\end{array}\right)_{1}\left(\begin{array}{c}
N_{-}+N_{+}-\mu \\
2 j^{\prime}+N_{+}+1-\mu
\end{array}\right\rangle_{1},
$$

where

$$
\sigma_{1}=2\left(J-j-j^{\prime}\right) N_{+}-N_{+}-N_{+}^{2}
$$

The second combinatorial coefficient can be transformed by using Eq. (2.9) in the following form:

$$
\left(\begin{array}{l}
A-\mu \\
B-\mu
\end{array}\right)_{1}=(-)^{B-\mu} q_{1}^{\phi}\left(\begin{array}{c}
B-A-1 \\
B-\mu
\end{array}\right)_{1}
$$

where

$$
\begin{gathered}
A=N_{-}+N_{+}=2 J, \\
B=2 j^{\prime}+N_{+}+1=2 j^{\prime}+J+j-j^{\prime}+1=J+\lambda+\lambda^{\prime}+1, \\
\phi=\mu^{2}-(4 J+1) \mu+2 A B+B-B^{2} .
\end{gathered}
$$

After this substitution Eq. (6.16) becomes

$$
I=q^{\sigma_{1}+\sigma_{2}} \sum_{\mu}(-)^{2 j^{\prime}} q^{-2\left(\mu^{2}-\mu\right)+4(j+J) \mu}\left\langle\begin{array}{c}
2 j^{\prime}-N_{-} \\
2 j^{\prime}+N_{+}+1-\mu
\end{array}\right\rangle_{1}\left\langle\begin{array}{c}
N_{+} \\
\mu
\end{array}\right\rangle_{1},
$$

where

$$
\sigma_{2}=B^{2}-2 A B-B
$$

Equation (6.20) is again of the form where Eq. (2.7) may be used to collapse the $\mu$-sum. That is,

$$
\sum_{\mu} q^{\theta}\left\langle\begin{array}{c}
N_{+} \\
\mu
\end{array}\right\rangle_{1}\left\langle\begin{array}{c}
2 j^{\prime}-N_{-} \\
2 j^{\prime}+N_{+}+1-\mu
\end{array}\right\rangle_{1}=\left\langle\begin{array}{c}
N_{+}-N_{-}+2 j^{\prime} \\
2 j^{\prime}+N_{+}+1
\end{array}\right\rangle_{1}
$$

where

$$
\theta=-2\left(\mu-N_{+}\right)\left(\mu-N_{+}-2 j^{\prime}-1\right)
$$

or

$$
\theta=-2\left(\mu^{2}-\mu\right)+4(j+J) \mu+\sigma_{3}, \quad \sigma_{3}=-2 N_{+}\left(2 j^{\prime}+N_{+}+1\right)
$$

and finally we get

$$
I=q^{\sigma_{1}+\sigma_{2}-\sigma_{3}(-)^{2 j^{\prime}}}\left(\begin{array}{c}
N_{+}-N_{-}+2 j^{\prime} \\
2 j^{\prime}+N_{+}+1
\end{array}\right\rangle_{1}
$$

Substituting into Eq. (6.14) one finds 


$$
\left|S_{j-j^{\prime}}^{J\left(j j^{\prime}\right)}\right|^{2}=(-)^{2 j^{\prime}} q^{\sigma_{4}}\langle 2 J+1\rangle_{1} \frac{\left\langle 2 j^{\prime}\right\rangle_{1} !\langle 2 j\rangle_{1} !\left\langle J-j-j^{\prime}-1\right\rangle_{1} !}{\left\langle J-j+j^{\prime}\right\rangle_{1} !\left\langle J+j+j^{\prime}+1\right\rangle_{1} !\left\langle j-j^{\prime}-J-1\right\rangle_{1} !}
$$

with

$$
\sigma_{4}=\sigma_{1}+\sigma_{2}-\sigma_{3}+2\left(J-j-j^{\prime}\right)=-2 j^{\prime}-4 j^{\prime} J+\dot{4} j^{\prime} j .
$$

In order to simplify Eq. (6.25) further, we can write a set of useful relations

$$
\frac{\left\langle J-j-j^{\prime}-1\right\rangle_{1} !}{\left\langle J-j+j^{\prime}\right\rangle_{1}}=\left(\begin{array}{c}
J-j-j^{\prime}-1 \\
-2 j^{\prime}-1
\end{array}\right\rangle_{1}\left\langle-2 j^{\prime}-1\right\rangle_{1} !
$$

and by Eq. (2.9)

$$
\left\langle\begin{array}{c}
J-j-j^{\prime}-1 \\
-2 j^{\prime}-1
\end{array}\right\rangle_{1}=(-)^{-2 j^{\prime}-1} q^{4 j^{\prime} J-4 j^{\prime} j+2 J-2 j}\left(\begin{array}{c}
j-J-j^{\prime}-1 \\
-2 j^{\prime}-1
\end{array}\right)_{1}
$$

Also

$$
\left\langle j-j^{\prime}-J-1\right\rangle_{1} !=\left\langle\begin{array}{c}
j-j^{\prime}-J-1 \\
-2 j^{\prime}-1
\end{array}\right\rangle_{1}\left\langle-2 j^{\prime}-1\right\rangle_{1} !\left\langle j+j^{\prime}-J\right\rangle_{1} !
$$

Using the relations $(6.26)-(6.28)$ we finally get

$$
\begin{gathered}
\left|S_{j-j^{\prime}}^{J\left(j j^{\prime}\right)}\right|^{2}=-q^{2\left(J-j-j^{\prime}\right)} \frac{\langle 2 J+1\rangle_{1}\left\langle 2 j^{\prime}\right\rangle_{1} !\langle 2 j\rangle_{1} !}{\left\langle J+j+j^{\prime}+1\right\rangle_{1} !\left\langle j+j^{\prime}-J\right\rangle_{1} !}, \\
S_{j-j^{\prime}}^{J\left(j j^{\prime}\right)}=i q^{J-j-j^{\prime}}\left[\frac{\langle 2 J+1\rangle_{1}\langle 2 j\rangle_{1} !\left\langle 2 j^{\prime}\right\rangle_{1} !}{\left\langle J+j+j^{\prime}+1\right\rangle_{1} !\left\langle j+j^{\prime}-J\right\rangle_{1} !}\right]^{1 / 2} .
\end{gathered}
$$

$S_{j-j^{\prime}}^{J\left(j j^{\prime}\right)}$ is usually chosen to be real. Then $S_{j-j^{\prime}}^{J\left(j j^{\prime}\right)}$ agrees with the Wigner result for $q=1$.

\section{GENERAL CASE (PART B)}

Having obtained $S_{j-j^{\prime}}^{J\left(j j^{\prime}\right)}$ for unrestricted $J$ we now compute $S_{\lambda \lambda^{\prime}}^{J\left(j j^{\prime}\right)}$, the corresponding most general Clebsch-Gordan coefficient, by use of Eq. (5.3)

$$
S_{\lambda \lambda^{\prime}}^{J\left(j j^{\prime}\right)}=\frac{[2 J+1]_{q} q^{2 \Lambda}}{S_{j-j^{\prime}}^{J\left(j j^{\prime}\right)}} h\left(D_{j \lambda}^{j} D_{-j^{\prime} \lambda^{\prime}}^{j^{\prime}} \bar{D}_{M, \Lambda}^{J}\right),
$$

where $M=j-j^{\prime}$ and $\Lambda=\lambda+\lambda^{\prime}$. One now has

$$
\begin{aligned}
& h\left(D_{j \lambda}^{j} D_{-j^{\prime} \lambda^{\prime}}^{j^{\prime}} \bar{D}_{M \Lambda}^{J}\right)=\left(\left\langle\begin{array}{c}
2 j \\
j+\lambda
\end{array}\right\rangle_{1}\left\langle\begin{array}{c}
2 j^{\prime} \\
j^{\prime}+\lambda^{\prime}
\end{array}\right\rangle_{1}\right)^{1 / 2} \Delta_{M \Lambda}^{J}\left(-q_{1}\right)^{j^{\prime}+\lambda^{\prime}} \sum\left\langle\begin{array}{c}
N_{+} \\
S
\end{array}\right\rangle\left\langle\begin{array}{c}
N_{-} \\
T
\end{array}\right\rangle q_{1}^{\left(N_{+}-S+1\right) T} \\
& \times(-)^{T} \delta\left(S+T, N_{+}^{\prime}\right) h\left[\left(a^{j+\lambda} b^{j-\lambda}\right)\left(\bar{b}^{j^{\prime}+\lambda^{\prime}} \bar{a}^{j^{\prime}-\lambda^{\prime}}\right)\left(a^{N_{-}-r_{b}} \bar{b}^{N_{+}-} S_{\bar{a}} S\right)\right] .
\end{aligned}
$$

By enforcing the delta function and using the commutation rules (3.2), the Woronowicz integral may again be reduced. One finds 


$$
\begin{aligned}
& h\left[\left(a^{j+\lambda} b^{j-\lambda}\right)\left(\bar{b}^{j^{\prime}+\lambda^{\prime}} \bar{a}^{j^{\prime}-\lambda^{\prime}}\right)\left(a^{N_{-}-T} b^{T} \bar{b}^{N_{+}-N_{+}^{\prime}+T} \bar{a}^{N_{+}^{\prime}-T}\right)\right] \\
& \quad=q^{\sigma_{1}+\sigma_{2}} h\left[\left(q_{1}^{2\left(N_{-}-N_{+}^{\prime}\right)} b \bar{b} \mid q^{2}\right)_{j^{\prime}-\lambda^{\prime}}\left(b \bar{b} \mid q^{2}\right)_{N_{+}^{\prime}-T}(b \bar{b})^{T+j-\lambda}\right],
\end{aligned}
$$

where

$$
\sigma_{1}=\left(j^{\prime}+\lambda^{\prime}+j-\lambda\right)(j+\lambda)+\left(N_{+}-N_{+}^{\prime}\right) N_{+}^{\prime}, \quad \sigma_{2}=\left(3 N_{+}^{\prime}-N_{+}-2 T\right) T .
$$

The reduced $h$ product (7.3) is again of the same form that was encountered before in Eqs. (4.15) and (6.3) and may be expanded in the same way. One then obtains

$$
\begin{aligned}
& h\left(\left(q_{1}^{2\left(N_{-}-N_{+}^{\prime}\right)} b \bar{b} \mid q^{2}\right)_{j^{\prime}-\lambda^{\prime}}\left(b \bar{b} \mid q^{2}\right)_{N_{+}^{\prime}-r}(b \bar{b})^{T+j-\lambda}\right) \\
& \quad=\sum_{\ell}(-)^{\ell} q^{2\left(j^{\prime}-\lambda^{\prime}\right) \ell} q^{-\ell^{2}-\ell}\left(\begin{array}{c}
j^{\prime}-\lambda^{\prime} \\
\ell
\end{array}\right)_{1}^{-2\left(N_{-}-N_{+}^{\prime}\right) \ell} B_{q}\left(T+j-\lambda+\ell+1, N_{+}^{\prime}-T\right) .
\end{aligned}
$$

By Eqs. (7.2), (7.3), and (7.5), and substituting the expression for $B_{q}$

$$
\begin{aligned}
h\left(D_{j \lambda}^{j} D_{-j^{\prime} \lambda^{\prime}}^{j^{\prime}} \bar{D}_{M \Lambda}^{J}\right)= & \eta \sum_{\ell}(-)^{\ell} q^{2\left(j+\lambda-N_{+}^{\prime}\right) \ell-\ell(\ell+1)}\left(\begin{array}{c}
j^{\prime}-\lambda^{\prime} \\
\ell
\end{array}\right\rangle_{1} \frac{1}{\left\langle j-\lambda+N_{+}^{\prime}+\ell+1\right\rangle_{1} !} \\
& \times \sum_{T}(-)^{T}\left(\begin{array}{c}
N_{+} \\
N_{+}^{\prime}-T
\end{array}\right\rangle_{1}\left\langle\begin{array}{c}
N_{-} \\
T
\end{array}\right\rangle_{1}\langle j-\lambda+t+\ell\rangle_{1} ! q^{T\left[2\left(j^{\prime}+\lambda^{\prime}+\ell\right)-T+1\right]},
\end{aligned}
$$

where

$$
\eta=\left(\left(\begin{array}{c}
2 j \\
j+\lambda
\end{array}\right)_{1}\left(\begin{array}{c}
2 j^{\prime} \\
j^{\prime}+\lambda^{\prime}
\end{array}\right)\right)_{1}^{1 / 2} \Delta_{M \Delta}^{J}\left(-q_{1}\right)^{j^{\prime}+\lambda^{\prime}} q^{\sigma_{1}-2(j-\lambda+1) N_{+}^{\prime}}
$$

Denote the $T$-sum by

$$
\sum_{T}=\sum_{T}(-)^{T}\left\langle\begin{array}{c}
N_{+} \\
N_{+}^{\prime}-T
\end{array}\right\rangle_{1}\left\langle\begin{array}{c}
N_{-} \\
T
\end{array}\right\rangle_{1}\left\langle N_{+}^{\prime}-T\right\rangle_{1} !\langle j-\lambda+T+\emptyset\rangle_{1} ! q^{T\left[2\left(j^{\prime}+\lambda^{\prime}+\varrho-T+1\right]\right.}
$$

Again set $t=N_{-}-T$. Then the sum in Eq. (7.8) becomes

$$
\sum_{T}=\eta_{1} \sum_{t}(-)^{-t}\left\{\begin{array}{c}
N_{-} \\
t
\end{array}\right\rangle_{1}\left(\begin{array}{c}
j-\lambda+N_{-}+\ell-t \\
N_{-}^{\prime}-t
\end{array}\right)_{1} q^{t\left[2\left(N_{-}-j^{\prime}-\lambda^{\prime}-\ell\right)-t-1\right]},
$$

where

$$
\eta_{1}=(-)^{N_{-}} q^{2 N_{-} \ell} q^{N_{-}\left(2 j^{\prime}+2 \lambda^{\prime}-N_{-}+1\right)}\left\langle N_{+}\right\rangle_{1} !\left\langle j^{\prime}+\lambda^{\prime}+\not\right\rangle_{1} !
$$

By Eq. (2.9) the second combinatorial factor in Eq. (7.9) may be transformed

$$
\left(\begin{array}{c}
j-\lambda+N_{-}+\ell-t \\
N_{-}^{\prime}-t
\end{array}\right)_{1}=(-)^{-N_{-}^{\prime}+t} q^{\phi}\left(\begin{array}{c}
N_{-}^{\prime}-j+\lambda-N_{-}-\ell-1 \\
N_{-}^{\prime}-t
\end{array}\right)_{1}
$$


where

$$
\phi=2\left(N_{-}^{\prime}-t\right)\left(N_{-}^{\prime}-j+\lambda-N_{-}-\ell-1\right)+\left(N_{-}^{\prime}-t\right)\left(1-N_{-}^{\prime}+t\right) .
$$

Then the $t$-sum becomes

$$
\sum_{t}=\eta_{2} \sum_{t}\left(\begin{array}{c}
N_{-}^{\prime}-j+\lambda-\ell-N_{-}-1 \\
N_{-}^{\prime}-t
\end{array}\right\rangle_{1}\left(\begin{array}{c}
N_{-} \\
t
\end{array}\right)_{1} q^{-2 t^{2}} q^{t\left[4 N_{-}-2\left(j^{\prime}+\lambda^{\prime}-j+\lambda\right)\right]}
$$

with

$$
\eta_{2}=(-)^{N_{-}-N_{-}^{\prime}} q^{2\left(N_{-}-N_{-}^{\prime}\right)} q^{N_{-}\left(2 j^{\prime}+2 \lambda^{\prime}-N_{-}+1\right)} q^{N_{-}^{\prime}\left(2\left(-j+\lambda-N_{-}\right)-1+N_{-}^{\prime}\right)}\left\langle N_{+}\right\rangle_{1} !\left\langle j^{\prime}+\lambda^{\prime}+\not\right\rangle_{1} ! .
$$

Equation (7.13) is of the form where Eq. (2.7) may be used to collapse the $t$-sum

$$
\sum_{t} q^{\theta}\left\langle\begin{array}{c}
N_{-}^{\prime}-j+\lambda-\ell-N_{-}-1 \\
N_{-}^{\prime}-t
\end{array}\right\rangle_{1}\left\langle\begin{array}{c}
N_{-} \\
t
\end{array}\right\rangle_{1}=\left\langle\begin{array}{c}
N_{-}^{\prime}-j+\lambda-\ell-1 \\
N_{-}^{\prime}
\end{array}\right\rangle_{1}
$$

where

$$
\theta=-2\left(N_{-}-t\right)\left(N_{-}^{\prime}-t\right)
$$

or

$$
\theta=-2 t^{2}+t\left[4 N_{-}-2\left(j^{\prime}+\lambda^{\prime}-j+\lambda\right)\right]+\sigma_{3}, \quad \sigma_{3}=-2 N_{-} N_{-}^{\prime}
$$

and we have

$$
\sum_{t}=\eta_{2} q^{-\sigma_{3}}\left(\begin{array}{c}
N_{-}^{\prime}-j+\lambda-\ell-1 \\
N_{-}^{\prime}
\end{array}\right)_{1}
$$

Substituting in Eq. (7.6), we find

$$
\begin{aligned}
h\left(D_{j \lambda}^{j} D_{-j^{\prime} \lambda^{\prime}}^{j^{\prime}} \vec{D}_{M \Lambda}^{J}\right)= & \eta \eta_{2} q^{-\sigma_{3}} \sum_{\ell}(-)^{\prime} q^{2\left(j+\lambda-N_{+}^{\prime}\right) \ell-\ell(\ell+1)} \\
& \times\left(\begin{array}{c}
j^{\prime}-\lambda^{\prime} \\
\ell
\end{array}\right\rangle_{1}\left(\begin{array}{c}
N_{-}^{\prime}-j+\lambda-\ell-1 \\
N_{-}^{\prime}
\end{array}\right\rangle_{1} \frac{1}{\left\langle j-\lambda+N_{+}^{\prime}+\ell+1\right\rangle_{1} !} .
\end{aligned}
$$

Using the expression (7.19) and writing explicitly $N_{ \pm}^{\prime}, N_{ \pm}$, and $S_{j-j^{\prime}}^{J\left(j j^{\prime}\right)}$ we finally obtain from Eq. (7.1)

$$
S_{\lambda \lambda^{\prime}}^{J\left(j j^{\prime}\right)}=M q^{\Phi} \sum_{\ell}(-)^{\ell} q^{\psi(\ell)}\left(\begin{array}{c}
j^{\prime}-\lambda^{\prime} \\
\ell
\end{array}\right)_{1} \frac{\left\langle j^{\prime}+\lambda^{\prime}+\ell\right\rangle_{1} !}{\left\langle J+j+\lambda^{\prime}+\ell+1\right\rangle_{1} !}\left(\begin{array}{c}
J-j-\lambda^{\prime}-\ell-1 \\
J-\lambda-\lambda^{\prime}
\end{array}\right)_{1},
$$

with

$$
M=(-)^{p}\left(\frac{\langle 2 J+1\rangle_{1}\langle J+\Lambda\rangle_{1} !\langle J-\Lambda\rangle_{1} !\left\langle J+j+j^{\prime}+1\right\rangle_{1} !\left\langle j+j^{\prime}-J\right\rangle_{1} !\left\langle J+j-j^{\prime}\right\rangle_{1} !}{\langle j+\lambda\rangle_{1} !\langle j-\lambda\rangle_{1} !\left\langle j^{\prime}+\lambda^{\prime}\right\rangle_{1} !\left\langle j^{\prime}-\lambda^{\prime}\right\rangle_{1} !\left\langle J-j+j^{\prime}\right\rangle_{1} !}\right)^{1 / 2}
$$




$$
\begin{gathered}
p=2 j^{\prime}+2 \lambda^{\prime}-j+\lambda, \\
\Phi=-J+j^{\prime}+\lambda+\left(j^{\prime}+\lambda^{\prime}+j-\lambda\right)\left(j^{\prime}+\lambda-J\right), \\
\psi(\ell)=\ell\left[2\left(j^{\prime}+\lambda-J\right)-\ell-1\right] .
\end{gathered}
$$

Let us compare this final expression with the result of Groza et al. (GKK) written in our notation:

$$
\begin{aligned}
{\left[\begin{array}{lll}
j & j^{\prime} & J \\
\lambda & \lambda^{\prime} & \Lambda
\end{array}\right]=} & M^{\prime} q^{\Phi^{\prime}} \sum(-1)^{\ell} q^{\psi^{\prime}(\ell)}\langle j-\lambda+\ell\rangle_{1} !\left\langle j^{\prime}+J+\lambda-\ell\right\rangle_{1} ! \\
& \times \frac{1}{\langle\ell\rangle_{1} !\langle J+\Lambda-\ell\rangle_{1} !\left\langle j^{\prime}-j+J-\ell\right\rangle_{1} !\left\langle j-j^{\prime}-\Lambda+\ell\right\rangle_{1} !}
\end{aligned}
$$

with

$$
\begin{gathered}
M^{\prime}=(-)^{p^{\prime}}\left(\frac{\langle 2 J+1\rangle_{1}\langle J+\Lambda\rangle_{1} !\langle J-\Lambda\rangle_{1} !\left\langle j+j^{\prime}-J\right\rangle_{1} !\left\langle j-j^{\prime}+J\right\rangle_{1} !\left\langle J-j+j^{\prime}\right\rangle_{1} !}{\langle j+\lambda\rangle_{1} !\langle j-\lambda\rangle_{1} !\left\langle j^{\prime}+\lambda^{\prime}\right\rangle_{1} !\left\langle j^{\prime}-\lambda^{\prime}\right\rangle_{1} !\left\langle j+j^{\prime}+J+1\right\rangle_{1} !}\right)^{1 / 2} \\
p^{\prime}=j^{\prime}+\lambda^{\prime} \\
\Phi^{\prime}=-j^{2}-j+J-j^{\prime} J+j j^{\prime}+j J-\left(j^{\prime}-j+J+\lambda^{\prime}+\lambda\right) \lambda^{\prime}-\lambda(J-j)+\lambda^{\prime}(j+1), \\
\psi^{\prime}(\ell)=\ell\left[2\left(J+\lambda^{\prime}-j\right)-\ell-1\right] .
\end{gathered}
$$

Note that to recast their answer in our notation we have used $q=q_{\mathrm{GKK}}^{-1 / 2}$.

There is the following identity implied by the equality of (7.20) and (7.24):

$$
\begin{aligned}
q^{\Phi} & \sum(-1)^{\ell} \frac{q^{\psi(\mathscr{C})}\left\langle j^{\prime}+\lambda^{\prime}+\ell\right\rangle_{1} !\left\langle J-j-\lambda^{\prime}-\ell-1\right\rangle_{1} !}{\langle\mathscr{\ell}\rangle_{1} !\left\langle j^{\prime}-\lambda^{\prime}-\ell\right\rangle_{1} !\left\langle J+j+\lambda^{\prime}+\mathscr{\ell}+1\right\rangle_{1} !\langle\lambda-j-\ell-1\rangle_{1} !} \\
= & (-1)^{p^{\prime}-p} \frac{\left\langle J+j^{\prime}-j\right\rangle_{1} !\langle J-\Lambda\rangle_{1} !}{\left\langle J+j^{\prime}+j+1\right\rangle_{1} !\left\langle j^{\prime}-\lambda^{\prime}\right\rangle_{1} !} q^{\Phi^{\prime}} \sum(-1)^{n} q^{\psi^{\prime}(n)} \\
& \times \frac{\langle j-\lambda+n\rangle_{1} !\left\langle j^{\prime}+J+\lambda-n\right\rangle_{1} !}{\langle n\rangle_{1} !\langle J+\Lambda-n\rangle_{1} !\left\langle j^{\prime}-j+J-n\right\rangle_{1} !\left\langle j-j^{\prime}-\Lambda+n\right\rangle_{1} !} .
\end{aligned}
$$

The identity displayed in Eq. (7.28) can be expressed in terms of $q$-hypergeometric functions. By using the following expressions:

$$
\begin{aligned}
{ }_{n+1} \phi_{n}\left(a_{1}, \ldots, a_{n+1} ; b_{1}, \ldots, b_{n} \mid q, z\right) & ={ }_{n+1} \phi_{n}\left(\begin{array}{c}
a_{1}, \ldots, a_{n+1} \\
b_{1}, \ldots, b_{n}
\end{array} \mid q, z\right) \\
& =\sum_{m=0}^{\infty} \frac{\left(a_{1} ; q\right)_{m} \cdots\left(a_{n+1} ; q\right)_{m}}{\left(b_{1} ; q\right)_{m} \cdots\left(b_{n} ; q\right)_{m}} \frac{z^{m}}{(q ; q)_{m}}
\end{aligned}
$$

and

$$
{ }_{n+1} \Phi_{n}\left(a_{1}, \ldots, a_{n+1} ; b_{1}, \ldots, b_{n} \mid q, z\right)={ }_{n+1} \phi_{n}\left(q^{a_{1}}, \ldots, q^{a_{n+1}} ; q^{b_{1}}, \ldots, q^{b_{n}} \mid q, z\right)
$$


We get

$$
\mathscr{H}_{3} \Phi_{2}\left(\begin{array}{c}
j-j^{\prime}-J,-J-\lambda-\lambda^{\prime}, j-\lambda+1 \\
-j^{\prime}-J-\lambda, j-j^{\prime}-\lambda-\lambda^{\prime}-1
\end{array} \mid q, q\right)={ }_{3} \Phi_{2}\left(\begin{array}{c}
j^{\prime}+\lambda^{\prime}+1,-j^{\prime}+\lambda^{\prime}, j-\lambda+1 \\
j+\lambda^{\prime}+J+2, j+\lambda^{\prime}-J+1
\end{array} \mid q, q\right),
$$

where

$$
\begin{aligned}
\mathscr{A}= & (-1)^{-j^{\prime}+j-J} q^{(1 / 2)\left[(j-J)(j-\lambda+1)-\lambda^{\prime}(1+\lambda)\right]} \\
& \times \frac{\left[J-j-j^{\prime}\right] !\left[J-\lambda-\lambda^{\prime}\right] !\left[j^{\prime}+J+\lambda\right] !\left[j+\lambda^{\prime}+J+1\right] !\left[j+\lambda^{\prime}-J\right] !}{\left[j+j^{\prime}+J+1\right] !\left[J+\lambda+\lambda^{\prime}\right] !\left[j^{\prime}+\lambda^{\prime}\right] !\left[j^{\prime}-j+J\right] !\left[j-j^{\prime}-\lambda-\lambda^{\prime}\right] !} .
\end{aligned}
$$

The expressions in Eqs. (7.20) and (7.24) have a quite different structure. They must be equivalent however and their equivalence represents a $q$-identity.

We have checked this equivalence for the following special cases:

$$
\left\langle\Lambda\left|S_{j, j^{\prime}}^{j+j^{\prime}}\right| \lambda, \lambda^{\prime}\right\rangle\left\langle\Lambda\left|S_{j, j^{\prime}}^{j-j^{\prime}}\right| \lambda, \lambda^{\prime}\right\rangle\left\langle j-j^{\prime}\left|S_{j, j^{\prime}}^{J}\right| j, j^{\prime}\right\rangle\left\langle\Lambda\left|S_{j, 1}^{J}\right| \lambda, \lambda^{\prime}\right\rangle,
$$

where $\Lambda=\lambda+\lambda^{\prime}$.

These checks give us confidence in the accuracy of our work but, of course, the asserted equivalence of the two expressions depends on the fact that the two different procedures calculate the same object.

\section{ALTERNATIVE PRESENTATION OF CLEBSCH-GORDAN COEFFICIENT FOR SU(2)}

Integration over the group space of $\mathrm{SU}(2)$ leads to an expression of the following form:

$$
\int \cos ^{2 a}\left(\frac{1}{2} \beta\right) \sin ^{2 b}\left(\frac{1}{2} \beta\right) d R=B(a+1, b+1) \int d R
$$

where $(\alpha \beta \gamma)$ is the usual notation for the Eulerian angles, $\int d R$ is the volume of the group space, and $B(a+1, b+1)$ is the beta function. On the other hand, if one performs a corresponding calculation for $\mathrm{SU}_{4}(2)$, then one encounters a $q$-beta function buried in a double sum such as (7.6). If one now puts $q=1$ and evaluates Eq. (7.6), there are two possibilities which differ in the order of summation. The results are different although they must of course be equivalent since both sums are finite. If one does the $T$-sum first, one gets

$$
S_{\lambda \lambda^{\prime}}^{J\left(j j^{\prime}\right)}=M \sum_{\ell}(-1)^{\ell}\left(\begin{array}{c}
j^{\prime}-\lambda^{\prime} \\
\ell
\end{array}\right) \frac{\left(j^{\prime}+\lambda^{\prime}+\ell\right) !}{\left(J+j+\lambda^{\prime}+\ell+1\right) !}\left(\begin{array}{c}
J-j-\lambda^{\prime}-\ell-1 \\
J-\lambda-\lambda^{\prime}
\end{array}\right)
$$

in terms of the usual binomial coefficients.

Let us now do the $\ell$-sum first by returning to Eq. (7.6) with $q=1$, and without substituting the explicit expression for the beta function:

$$
h\left(D_{j \lambda}^{j} D_{-j^{\prime} \lambda^{\prime}}^{j^{\prime}} \bar{D}_{M \Lambda}^{J}\right)=\eta_{0} \sum_{T}(-1)^{T}\left(\begin{array}{c}
N_{+} \\
N_{+}^{\prime}-T
\end{array}\right)\left(\begin{array}{c}
N_{-} \\
T
\end{array}\right) \sum_{\ell}(-)^{\ell}\left(\begin{array}{c}
m \\
\ell
\end{array}\right) B\left(k+T+1, N_{+}^{\prime}-T+1\right),
$$

where

$$
k=\ell+j-\lambda, \quad m=j^{\prime}-\lambda^{\prime}, \quad \eta_{0}=\eta(q=1) .
$$


The $\ell$-sum is

$$
\sum(-1)^{\ell}\left(\begin{array}{l}
m \\
\ell
\end{array}\right) B\left(k+T+1, N_{+}^{\prime}-T+1\right)=\left(N_{+}^{\prime}-T\right) !(A-B) ! \sum_{\ell}(-)^{\ell}\left(\begin{array}{c}
\ell+A \\
\ell+B
\end{array}\right)\left(\begin{array}{l}
m \\
\ell
\end{array}\right)
$$

where

$$
\begin{gathered}
A=j-\lambda+T, \\
B=j-\lambda+N_{+}^{\prime}+1 .
\end{gathered}
$$

Let $r=m-\ell$, then

$$
\begin{aligned}
\sum(-1)^{f}\left(\begin{array}{c}
\ell+A \\
\ell+B
\end{array}\right)\left(\begin{array}{l}
m \\
\ell
\end{array}\right)=(-1)^{m} \sum(-1)^{-r}\left(\begin{array}{c}
A+m-r \\
B+m-r
\end{array}\right)\left(\begin{array}{c}
m \\
r
\end{array}\right) & =(-1)^{-B} \sum\left(\begin{array}{c}
B-A-1 \\
B+m-r
\end{array}\right)\left(\begin{array}{c}
m \\
r
\end{array}\right) \\
& =(-1)^{-B}\left(\begin{array}{c}
m+B-A-1 \\
B+m
\end{array}\right)
\end{aligned}
$$

By Eqs. (8.4) and (8.8)

$$
\sum(-1)^{f}\left(\begin{array}{c}
m \\
\ell
\end{array}\right) B\left(k+T+1, N_{+}^{\prime}-T+1\right)=\left(N_{+}^{\prime}-T\right) !(A-B) !(-1)^{B}\left(\begin{array}{c}
m+B-A-1 \\
B+m
\end{array}\right)
$$

and by Eq. (8.3)

$$
\begin{aligned}
h\left(D_{j \lambda}^{j} D_{j^{\prime}-\lambda}^{j^{\prime}}, \bar{D}_{M \Lambda}^{J}\right)= & \eta_{0}(-1)^{-B} N_{+} ! N_{-} ! \sum(-1)^{T} \frac{1}{\left(N_{+}-N_{+}^{\prime}+T\right) ! T !\left(N_{-}-T\right) !} \\
& \times \frac{(A-B) !\left(m+N_{+}^{\prime}-T\right) !}{\left(N_{+}^{\prime}-T-B\right) !(m+B) !} .
\end{aligned}
$$

But

$$
\frac{(A-B) !}{\left(N_{+}^{\prime}-T-B\right) !}=\frac{\left(-\left(N_{+}^{\prime}-T\right)-1\right) !}{(-A-1) !}=\frac{A !}{\left(N_{+}^{\prime}-T\right) !}
$$

by Eq. (2.9) with $q=1$.

By making the appropriate substitutions one finds

$$
S_{\lambda \lambda^{\prime}}^{J\left(j^{\prime}\right)}=M^{\prime}(q=1)(-1)^{-B} \sum_{T} \frac{(-1)^{T}(j-\lambda+T) !\left(j^{\prime}+J+\lambda-T\right) !}{T !(J+\Lambda-T) !\left(j^{\prime}-j+J-T\right) !\left(j-j^{\prime}-\Lambda+T\right) !},
$$

which except for the phase $(-1)^{-B}$ is the usual form given for SU(2). The identity implied by the equivalence of Eqs. (8.12) and (8.2) is 


$$
\begin{aligned}
\sum & (-1)^{\ell} \frac{\left(j^{\prime}+\lambda^{\prime}+\ell\right) !\left(J-j-\lambda^{\prime}-\ell-1\right) !}{\ell !\left(j^{\prime}-\lambda^{\prime}-\ell\right) !\left(J+j+\lambda^{\prime}+\ell+1\right) !(\lambda-j-\ell-1) !} \\
= & (-1)^{p^{\prime}-p-B} \frac{\left(J+j^{\prime}-j\right) !(J-\Lambda) !}{\left(J+j+j^{\prime}+1\right) !\left(j^{\prime}-\lambda^{\prime}\right) !} \sum(-1)^{T} \\
& \times \frac{(j-\lambda+T) !\left(j^{\prime}+J+\lambda-T\right) !}{(J+\Lambda-T) !\left(j^{\prime}-j+J-T\right) !\left(j-j^{\prime}-\Lambda+T\right) !} .
\end{aligned}
$$

Equation (8.2) is the $q=1$ limit of Eq. (7.20) and Eq. (8.12) is the $q=1$ limit of Eq. (7.24) cxcept for the additional phase $(-1)^{-B}$. Thus one of the two equivalent representations corresponds to carrying out the $T$-sum and the other carrying out the $\ell$-sum. We do not have an independent way of doing the $\ell$-sum when $q \neq 1$.

\section{INTEGRAL OF A MULTIPLE PRODUCT ON q-GROUP SPACE}

The Schur orthogonality relations fix the value of the Woronowicz integral: $h\left(D_{\mu \lambda}^{j} \bar{D}_{\mu^{\prime} \lambda^{\prime}}^{j^{\prime}}\right)$. We may now calculate the integral of the triple product: $h\left(D_{\mu \lambda}^{j} D_{\mu^{\prime} \lambda^{\prime}}^{j^{\prime}} \bar{D}_{M \Lambda}^{J}\right)$.

We have already calculated the special cases

$$
\begin{array}{ll}
h\left(D_{\mu \lambda}^{j} D_{\mu^{\prime} \lambda^{\prime}}^{j^{\prime}} \bar{D}_{\mu+\mu^{\prime}, \lambda+\lambda^{\prime}}^{j+j^{\prime}}\right), & h\left(D_{j j}^{j} D_{-j^{\prime},-j^{\prime}}^{j^{\prime}} \bar{D}_{j-j^{\prime}, j-j^{\prime}}^{J}\right), \\
h\left(D_{\mu \lambda}^{j} D_{\mu^{\prime} \lambda^{\prime}}^{j^{\prime}} \bar{D}_{\mu+\mu^{\prime}, \lambda+\lambda^{\prime}}^{j-j^{\prime}}\right), & h\left(D_{j \lambda}^{j} D_{-j^{\prime} \lambda^{\prime}}^{j^{\prime}} \bar{D}_{j-j^{\prime}, \lambda+\lambda^{\prime}}^{J}\right) .
\end{array}
$$

The general result is now given by Eqs. (5.3) and (7.20)

$$
h\left(D_{\mu \lambda}^{j} D_{\mu^{\prime} \lambda^{\prime}}^{j^{\prime}} \bar{D}_{\mu \Lambda}^{J}\right)=\left(M\left|S^{J}\right| \mu \mu^{\prime}\right)\left(\Lambda\left|S^{J}\right| \lambda \lambda^{\prime}\right) q_{1}^{2 \Lambda} /[2 J+1]_{q} \delta\left(\mu+\mu^{\prime}, M\right) \delta\left(\lambda+\lambda^{\prime}, \Lambda\right) .
$$

The integral in Eq. (9.2) may be put in a symmetric form by replacing the third factor $\bar{D}_{\mu \Lambda}^{J}$. To do this we introduce the matrix $C$ which intertwines $D$ and $\bar{D}$ (Ref. 7) and corresponds to the usual charge conjugation operator

$$
\begin{gathered}
D=C^{-1} \bar{D} C, \\
C_{n m}=(-q)^{n} \delta(n+m, 0), \\
C^{2}=1 .
\end{gathered}
$$

Then

$$
D_{P Q}=\sum C_{P N} \bar{D}_{N M} C_{M Q}
$$

By Eq. (9.2)

$$
\begin{aligned}
h\left(D_{n_{1} m_{1}}^{j_{1}} D_{n_{2} m_{2}}^{j_{2}} D_{P Q}^{J}\right)= & \sum C_{P N}\left(N\left|S^{J}\right| n_{1} n_{2}\right) C_{M Q}\left(M\left|S^{J}\right| m_{1} m_{2}\right) q_{1}^{2 M} /[2 J+1]_{q} \\
& \times \delta\left(n_{1}+n_{2}, N\right) \delta\left(m_{1}+m_{2}, M\right) \\
= & \left(-q_{1}\right)^{J-P}\left(-q_{1}\right)^{J-Q}\left(-P\left|S^{J}\right| n_{1} n_{2}\right)\left(-Q\left|S^{J}\right| m_{1} m_{2}\right) /\langle 2 J+1\rangle_{1} .
\end{aligned}
$$

In obtaining Eq. (9.8) we have used the fact that $J-Q$ is an integer. Define a $q, 3 j$-symbol by 


$$
\left[\begin{array}{lll}
j_{1} & j_{2} & j_{3} \\
m_{1} & m_{2} & m_{3}
\end{array}\right]=\left(-q_{1}\right)^{j_{3}-m_{3}}\left(-m_{3}\left|S^{j_{3}}\right| m_{1} m_{2}\right) /\left\langle 2 j_{3}+1\right\rangle^{1 / 2}
$$

Then the integral of the triple product is

$$
h\left(D_{n_{1} m_{1}}^{j_{1}} D_{n_{2} m_{2}}^{j_{2}} D_{n_{3} m_{3}}^{j_{3}}\right)=\left[\begin{array}{ccc}
j_{1} & j_{2} & j_{3} \\
n_{1} & n_{2} & n_{3}
\end{array}\right]\left[\begin{array}{ccc}
j_{1} & j_{2} & j_{3} \\
m_{1} & m_{2} & m_{3}
\end{array}\right] .
$$

By successive application of Eq. (5.2) one may convert the Woronowicz integral of a general product such as $h\left(D_{n_{1} m_{1}}^{j_{1}} D_{n_{2} m_{2}}^{j_{2}} \cdots D_{n_{s} m_{s}}^{j_{s}}\right)$ into a multinomial in the $3 j$-symbols.

Finally, note

$$
h\left(D_{m_{1} m_{1}}^{j_{1}} D_{m_{2} m_{2}}^{j_{2}} D_{m_{3} m_{3}}^{j_{3}}\right)=\left[\begin{array}{ccc}
j_{1} & j_{2} & j_{3} \\
m_{1} & m_{2} & m_{3}
\end{array}\right]^{2}
$$

The permutation symmetries of the left side obviously also hold for the square of the $3 j$-symbol. This equation may be used in determining the symmetries of the $3 j$-symbols.

\section{FINAL REMARKS}

Relation (7.31), here established by comparing our expression for the Clebsch-Gordan coefficients with a differently derived result, is also a consequence of the symmetry properties of $q$-hypergeometric functions and can be proven by repeated applications of the Sears relation. ${ }^{8}$ Similarly expression (8.13) obtained in the limit $q \rightarrow 1$ is a consequence of the symmetry properties of the ${ }_{3} F_{2}$ hypergeometric function of unit argument. ${ }^{9}$ In Sec. IX we establish a relation between the integral over the group space of three matrix elements of irreducible representations and the square of $3 j$-symbols for $\mathrm{SU}_{q}(2)$. The permutation symmetries of the integral expression that are reflected in the permutation symmetries of the $3 j$-symbols could also be derived by expressing the Clebsch-Gordan coefficients in terms of the $q$-hypergeometric functions. It was also pointed out to us that similar integrals and their relations to Clebsch-Gordan coefficients had previously been considered in Ref. 10, but they had not been evaluated explicitly. Finally some of the relations of Sec. III and summation formulas in Sec. IV are well established in the literature ${ }^{2,3,8,11}$ and are given here for the sake of completeness and to establish the convention to be followed in the rest of the article.

\section{ACKNOWLEDGMENTS}

One of us (A.C.C.) thanks the TEP group at UCLA for their continuous hospitality. The authors want to acknowledge some useful comments by the referee, particularly in connection with the use of the Sears relation.

'A. N. Kirilov and N. Ju. Reshetikhin, in New Developments in the Theory of Knots, edited by T. Kohno (World Scientific, Singapore, 1990).

${ }^{2}$ V. A. Groza, I. I. Kachurik, and A. U. Klimyk, J. Math. Phys. 31, 2769 (1990).

${ }^{3}$ H. T. Koelink and T. H. Koorwinder, Proc. K. Ned. Akad. Wet. A 92, 443 (1989).

${ }^{4}$ E. R. Wigner, Group Theory and Its Application to the Quantum Mechanics of Atomic Spectra (Academic, New York, 1959).

'S. L. Woronowicz, Commun. Math. Phys. 111, 613 (1987). S. L. Woronowicz, RIMS Kyoto 23, 112 (1987).

${ }^{6}$ A. C. Cadavid and R. J. Finkelstein, UCLN/94/TEP/19, and Lett. Math. Phys. (to appear).

${ }^{7}$ T. H. Koornwinder, in Orthogonal Polynomials in Connection with Quantum Groups, edited by P. Nevai, NATO ASI, Series C, Vol. 294.

${ }^{8}$ G. Gasper and M. Rahman, Basic Hypergeometric Series (Cambridge University, New York, 1990).

${ }^{9}$ N. Ja. Vilenkin and A. U. Klimyk, Representation of Lie Groups and Special Functions, Volume 1: Simplest Lie Groups, Special Functions, and Integral Transforms (Kluwer Academic, The Netherlands, 1991).

${ }^{10} \mathrm{~N}$. Ja. Vilenkin and A. U. Klimyk, Representation of Lie Groups and Special Functions, Volume 3: Classical and Quantum Groups and Special Functions (Kluwer Academic, The Netherlands, 1991).

"T. Masuda, K. Mimachi, Y. Nagakami, M. Noumi, and K. Ueno, J. Functional Anal. 99, 357 (1991). 